\title{
Economic burden of maintenance hemodialysis patients' families in Nanchong and its influencing factors
}

\author{
Yunyan $\mathrm{Ma}^{1}$, Hang $\mathrm{Yu}^{2}$, Hongbing $\mathrm{Sun}^{3}, \mathrm{Mi} \mathrm{Li}^{4}, \mathrm{Li} \mathrm{Li}^{5}, \mathrm{Meng} \mathrm{Qin}^{1}$ \\ ${ }^{1}$ Blood Purification Room of Nephrology Department, Affiliated Hospital of North Sichuan Medical College, Nanchong, China; ${ }^{2}$ Ministry of Cadre \\ Health Care, Affiliated Hospital of North Sichuan Medical College, Nanchong, China; ${ }^{3}$ Department of Rheumatology and Immunology, Nanchong \\ Central Hospital of Sichuan Province, Nanchong, China; ${ }^{4}$ Blood Purification Center of Nephrology Department, Nanchong Central Hospital of \\ Sichuan Province, Nanchong, China; ${ }^{5}$ Hemodialysis Center, People's Hospital of Gaoping District, Nanchong, China \\ Contributions: (I) Conception and design: Y Ma, M Qin; (II) Administrative support: H Yu; (III) Provision of study materials or patients: Y Ma, H Yu, \\ H Sun, M Li, L Li; (IV) Collection and assembly of data: All authors; (V) Data analysis and interpretation: Y Ma, H Sun, M Li, L Li, M Qin; (VI) \\ Manuscript writing: All authors; (VII) Final approval of manuscript: All authors. \\ Correspondence to: Meng Qin. Blood Purification Room of Nephrology Department, Affiliated Hospital of North Sichuan Medical College, 63 \\ Wenhua Road, Shunqing District, Nanchong 637000, China. Email: qinmo0705@163.com.
}

Background Maintenance hemodialysis is the main therapy for clinical treatment of end-stage renal disease (ESRD). The aim of this study was to analyze the current status of the economic burden levied on families with members who are maintenance hemodialysis patients in Nanchong, and the related influencing factors.

Methods: A total of 111 patients with ESRD who were admitted to our hospital from April 2018 to April 2020 and treated with maintenance hemodialysis were selected as research subjects. A questionnaire survey was adopted as a data collection and interview method to observe the economic burden of families with a member who was a maintenance hemodialysis patient. Logistic regression analysis was used to analyze the independent risk factors that affect this economic burden.

Results: The direct economic burden, indirect economic burden, and average annual total economic burden (the sum of the direct economic burden and indirect economic burden of hemodialysis patients) of patients in the resident medical insurance group were significantly higher than those in the employee medical insurance group, resident medical insurance + poverty relief group, and employee medical insurance + poverty relief group $(\mathrm{P}<0.05)$. The analysis of the unconditional multifactor logistic regression model showed that age, occupation, monthly family income, and medical insurance type were independent risk factors that affected the average annual total economic burden of patients with maintenance hemodialysis $(\mathrm{P}<0.01)$.

Conclusions: Various medical insurance systems can effectively reduce the economic burden of hemodialysis patients, but patients must still bear significant financial hardship. It is necessary to further improve the medical insurance for patients with hemodialysis and increase management efforts to popularize the poverty relief policy.

Keywords: Maintenance hemodialysis; economic burden; influencing factors

Submitted Aug 17, 2020. Accepted for publication Oct 24, 2020.

doi: 10.21037/apm-20-1787

View this article at: http://dx.doi.org/10.21037/apm-20-1787

\section{Introduction}

Hemodialysis refers to the process of taking the patient's blood out of the body; exchanging substances between the blood and dialysate in the dialyzer to expel waste, excess water, and correct electrolyte and acid-base balance disorders; and then returning the blood to the body 
$(1,2)$. In recent years, more kinds of diseases have been found to potentially lead to chronic renal failure, and the incidence of chronic renal failure and acute renal failure is also increasing (3). Maintenance hemodialysis is the main method for clinical treatment of end-stage renal disease (ESRD), and it can effectively remove metabolic waste or toxins from the patient's body; correct the imbalance of water, electrolyte, and acid-base; normalize the internal environment; and prolong the patient's survival period $(4,5)$. However, patients with ESRD require life-long treatment, which incurs a serious economic burden on the patient's family and society. At present, although hemodialysis cases are regarded as special diseases in most areas, and a greater degree of poverty relief policy is given to these patients, the financial cost of this treatment is still considerably onerous. To examine this matter further, this present study enrolled 111 patients undergoing maintenance hemodialysis in Nanchong, Sichuan, China, as the research objects. The status of the economic burden the patients' families needed to bear was investigated, and the related risk factors were analyzed. We present the following article in accordance with the MDAR reporting checklist (available at http:// dx.doi.org/10.21037/apm-20-1787).

\section{Methods}

\section{General information}

A total of 111 patients with ESRD who received maintenance hemodialysis in our hospital from April 2018 to April 2020 were selected as the research subjects. The inclusion criteria for subjects were the following: (I) aged $\geq 20$ years old, without mental illness; (II) in stable condition and willing to cooperate with the investigation; (III) no missing or lost clinical data; (IV) without disorders affecting conscious, and with the ability to communicate. Exclusion criteria for subjects included the following: (I) with severe gastrointestinal disease; (II) with malignant masses, severe infections, or other severe disorders; (III) unconscious and unable to successfully complete the questionnaire; (IV) on regular dialysis for fewer than 3 months; $(V)$ patients with chronic diseases such as hypertension and coronary heart disease. This study was approved by Affiliated Hospital of North Sichuan Medical College (No. 2020ER(N)050-1). All procedures performed in this study involving human participants were in accordance with the Declaration of Helsinki (as revised in 2013). Informed consent was taken from all the patients.

\section{Questionnaire survey}

A questionnaire survey was performed and adopted a combined data collection and interview method. For the included maintenance hemodialysis patients, the professionally trained investigators performed the questionnaire surveys on the dialysis patients or their families with the assistance of clinicians or nurses. The main content of the survey was as follows: (I) general situation questionnaire, which included the patients' age, gender, occupation, education level, monthly family income per capita, and medical insurance type; (II) economic burden, which included the direct economic burden (the total annual cost of disease prevention, such as the costs of drugs, laboratory tests, hospitalization, hemodialysis) and the indirect economic burden (the social costs lost by illness, disability, and death, including expenses for escort, transportation, nutrition, and missing work of family members) (6).

\section{Observation indicators}

The current status of the economic burden of families with members on maintenance hemodialysis was observed, and logistic regression analysis was employed to analyze the independent risk factors that affect the increase in the economic burden of maintenance hemodialysis patients.

\section{Statistical methods}

Data in this study were analyzed using the SPSS 22.0 software (IBM, Armonk, NY, USA). Measurement data are described as mean \pm standard deviation $\left(\bar{x}_{ \pm} \mathrm{S}\right)$ and were analyzed using a $t$ test or analysis of variance. Measurement data are described as relative numbers including as ratios or composition ratios. Multiple logistic regression analysis, with the test level of $\alpha=0.05$, was performed to analyze the risk factors that affect the increasing economic burden of maintenance hemodialysis patients.

\section{Results}

Comparison of the direct economic burden of maintenance hemodialysis patients in each group

Among the 111 patients, 70 were males and 41 were females, aged from 25 to 77 years old, with an average age of $46.97 \pm 7.51$ years old. The dialysis age was from 2 to 
Table 1 Comparison of the direct economic burden of maintenance hemodialysis patients in each group $\left(\bar{x}_{ \pm} \mathrm{S}, \mathrm{CNY}\right)$ in terms of hospital bed charges, temperature adjustment fees, examination fees, consultation fees, treatment fees, injection fees, blood transfusion fees, surgery fees, oxygen transfer fees, radiation fees, hyperbaric oxygen treatment fees, and graphic printing fees

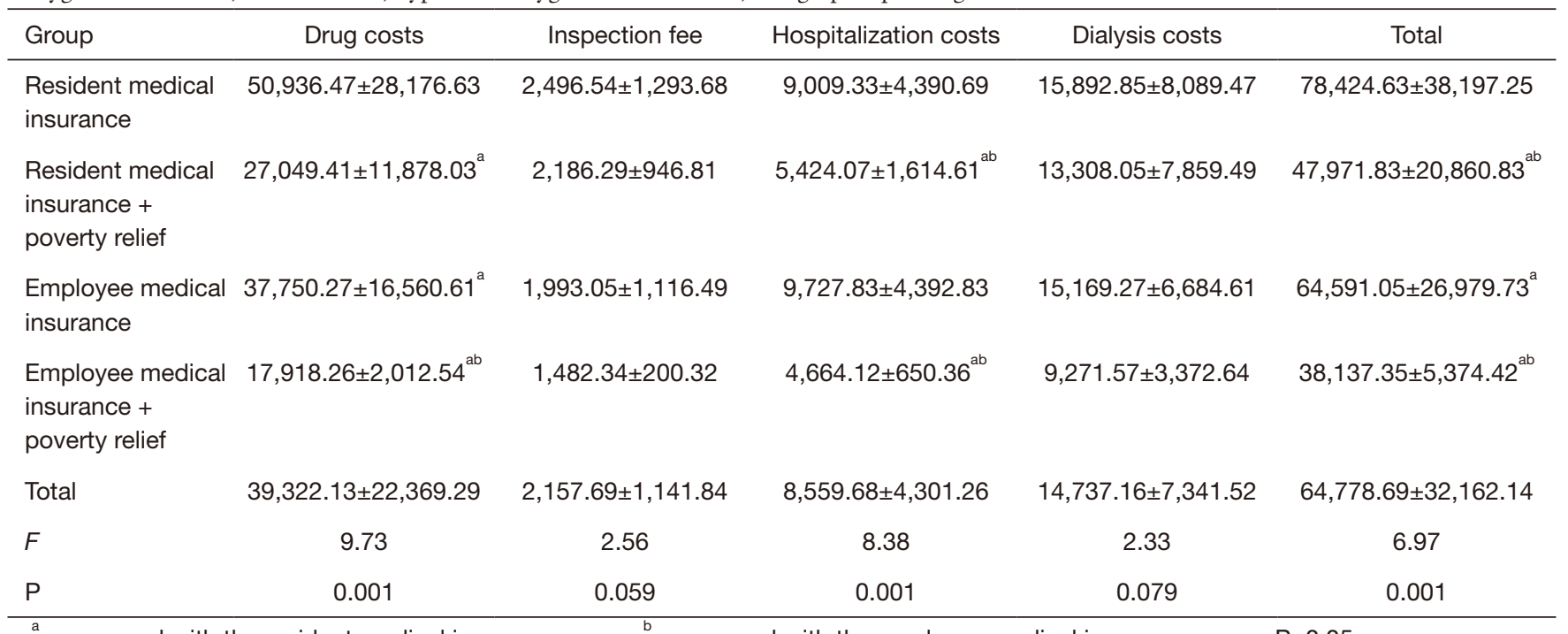

${ }^{\mathrm{a}}$, compared with the resident medical insurance group; ${ }^{\mathrm{b}}$, compared with the employee medical insurance group. $\mathrm{P}<0.05$.

9 years, with an average age of 6.14 years. Types of medical insurance included 36 cases with resident medical insurance, 18 cases with resident medical insurance + poverty relief, 48 cases of employee medical insurance, and 9 cases of employee medical insurance + poverty relief.

The cost of drugs in the resident medical insurance group was significantly higher than that of each of the three groups including the resident medical insurance + poverty relief group, the employee medical insurance group, and the employee medical insurance + the poverty relief group. Furthermore, the costs of drugs in the employee medical insurance + poverty relief group was the lowest, followed by the employee medical insurance group, and the resident medical insurance + poverty relief group. The hospitalization expense was the highest in the employee medical insurance group, followed by the resident medical insurance group, the resident medical insurance + poverty relief group, and the employee medical insurance + poverty relief group from, and the difference between these groups was statistically significant $(\mathrm{P}<0.05)$. There was no significant difference in the costs of chemotherapy and dialysis $(\mathrm{P}>0.05$, Table 1).

\section{Comparison of direct and indirect medical expenses of maintenance hemodialysis patients in each group}

The direct economic burden, indirect economic burden, and average annual total economic burden (the sum of the direct economic burden and indirect economic burden of hemodialysis patients) in the resident medical insurance group were significantly higher than those of other three groups (Figure 1), and the difference between the groups was statistically significant $(\mathrm{P}<0.05$, Table 2).

\section{Single-factor analysis of risk factors of the average annual total economic burden of patients}

The average annual economic burden of 111 patients with maintenance hemodialysis was tested for normal distribution. The results showed that there were significant differences in the average annual economic burden of patients of different ages, occupations, family monthly incomes, and medical insurance types $(\mathrm{P}<0.05$, Table 3$)$.

\section{Multivariate analysis of factors affecting the average annual total economic burden of patients}

According to the analysis of the unconditional multifactor logistic regression model, the results showed that age $>50$, being unemployed, a monthly family income of less than $¥ 3,000 \mathrm{CNY}$, and resident medical insurance were independent risk factors that adversely affected the average annual total economic burden of maintenance hemodialysis patients $(\mathrm{P}<0.01$, Table 4). 


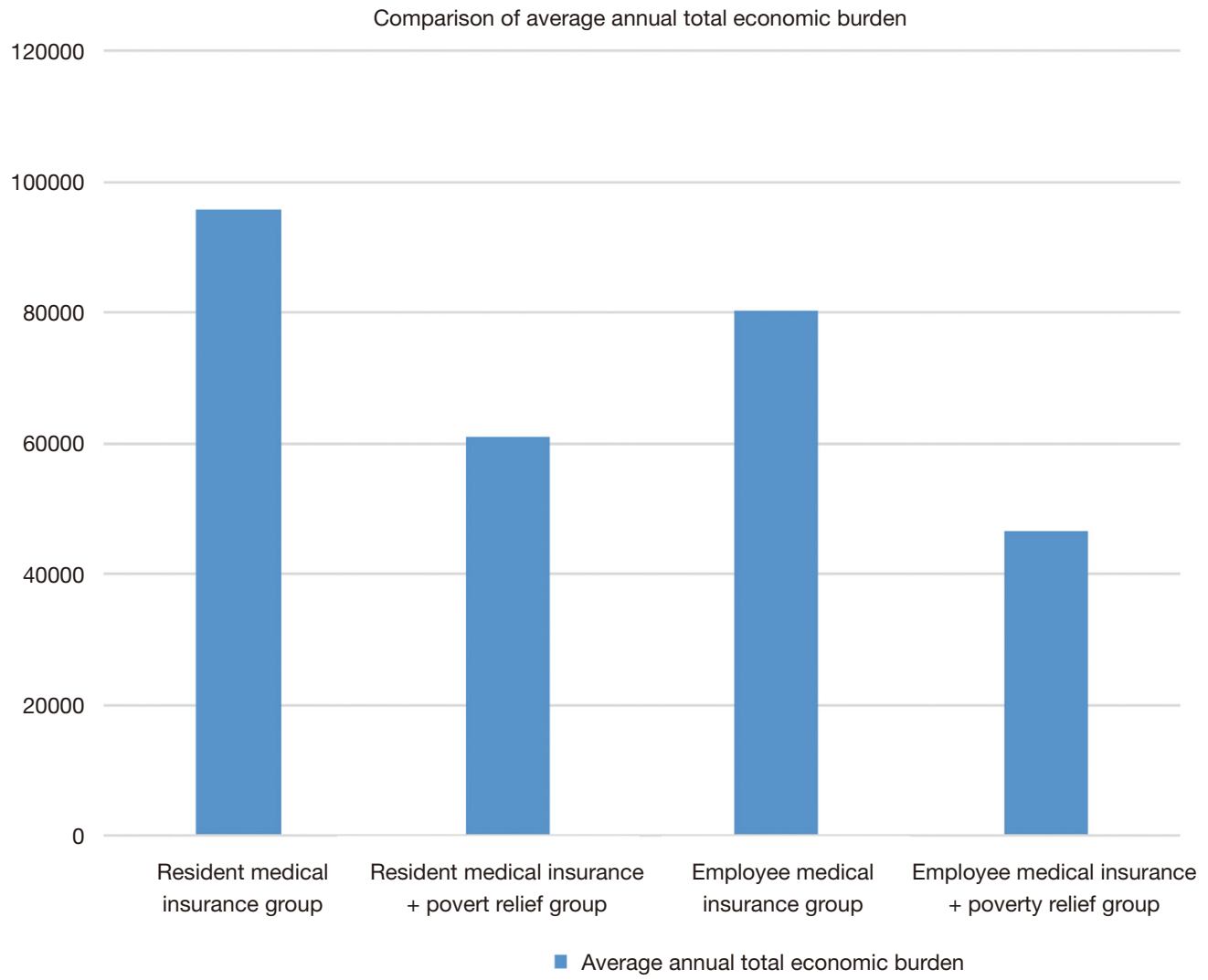

Figure 1 Comparison of the average annual total economic burden among the groups.

Table 2 Comparison of direct and indirect medical expenses of maintenance hemodialysis patients in each group $(\bar{x} \pm \mathrm{S}, \mathrm{CNY})$

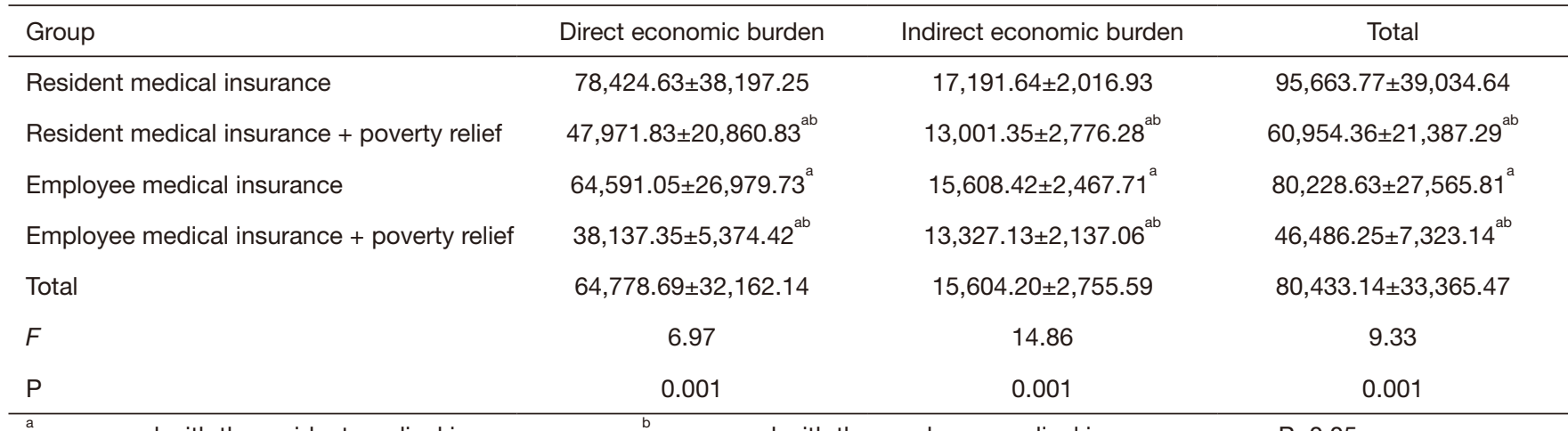

${ }^{a}$, compared with the resident medical insurance group; ${ }^{b}$, compared with the employee medical insurance group. $P<0.05$.

\section{Discussion}

Maintenance hemodialysis is a commonly used method for the treatment of renal failure and chronic kidney disease. This treatment method has the advantages of delaying the disease progression of patients and prolonging their survival time, so it has been widely accepted by clinicians and patients. Generally, maintenance hemodialysis patients receive hemodialysis treatment in the outpatient department. However, a series of complications may occur in some patients, and if the complications cannot be solved by the outpatient department, hospitalization are required. In addition, maintenance hemodialysis patients are at 
Table 3 Single-factor analysis of influencing factors of patients' average annual total economic burden $\left(\bar{x}_{ \pm} \mathrm{S}, \mathrm{CNY}\right)$

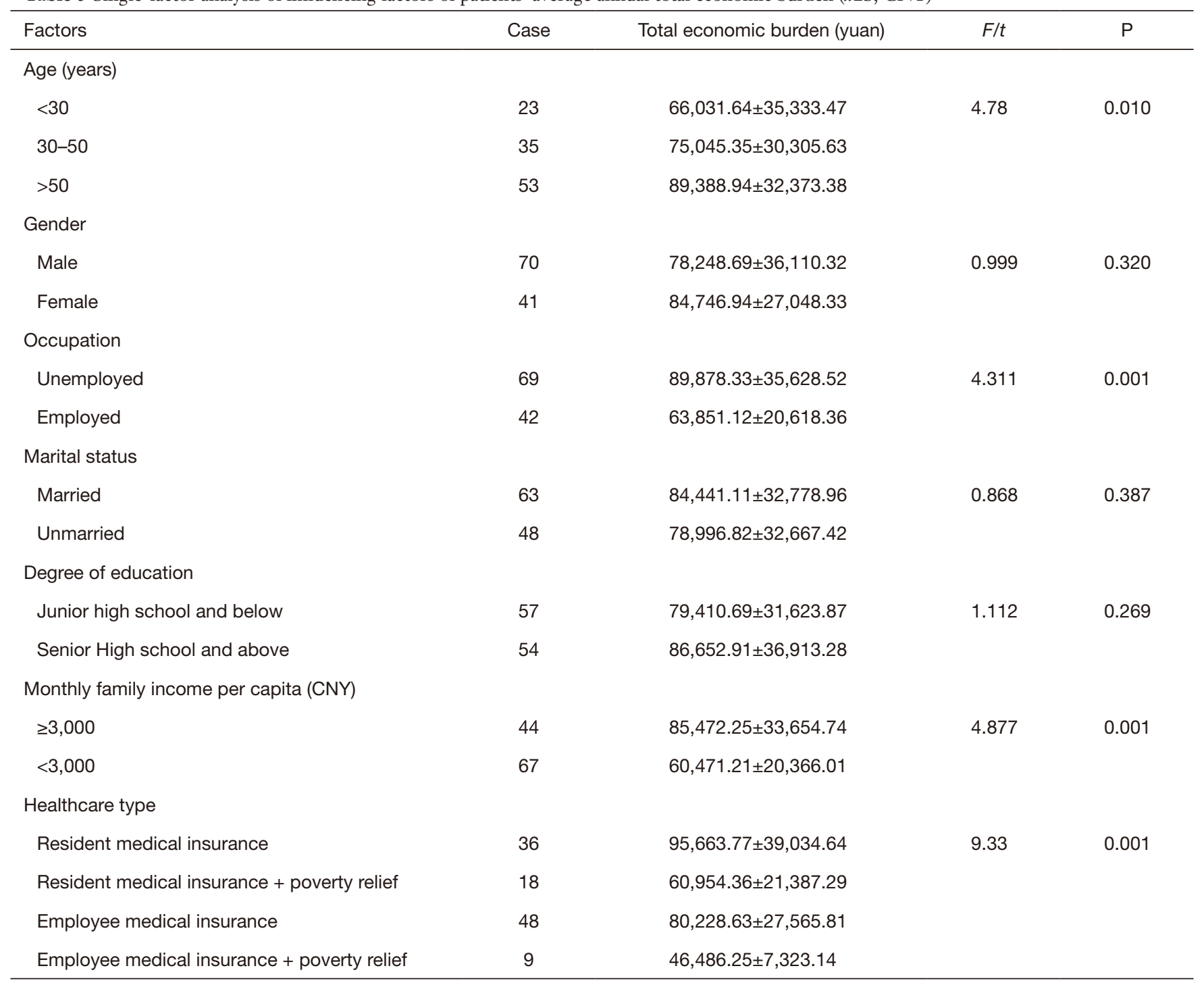

Table 4 Analysis of multiple factors influencing the treatment compliance of patients with maintenance hemodialysis

\begin{tabular}{lccccc}
\hline Factor & Regression coefficient & SEM & Wald $\chi^{2}$ & $P$ & OR (95\% Cl) \\
\hline Age $>50$ years & 0.587 & 0.221 & 8.642 & $<0.01$ & $1.799(1.166-2.774)$ \\
Unemployed & 0.625 & 0.234 & 10.3425 & $<0.01$ & $1.868(1.181-2.955)$ \\
Monthly family income per capita $<3,000$ CNY & 0.659 & 0.211 & 12.526 & $<0.01$ & $1.933(1.278-2.923)$ \\
Resident medical insurance & 0.851 & 0.340 & 9.327 & $<0.01$ & $2.342(1.203-4.560)$ \\
\hline
\end{tabular}

SEM, standard error of mean.

high risk of nosocomial infection. According to relevant data, more than $50 \%$ of patients undergoing hemodialysis will suffer from malnutrition, complicated with acute and chronic infections and other phenomena, which will seriously affect the quality of life and survival time of patients and increase their economic burden (6). 


\section{The significant economic burden of maintenance hemodialysis patient families}

The results in this study showed that maintenance hemodialysis levies a massive economic burden upon patients. Drug fee accounted the highest portion of costs, with an average annual expense of $¥ 50,936.47 \pm 28,176.63$ CNY, followed by dialysis, laboratory tests, and hospitalization costs. It has been pointed out that the costs of drug treatment for maintenance hemodialysis patients is high, accounting for about $50 \%$ of the total economic burden of patients (7). With the continuous application of new technologies and drugs, the increase in the number of patients and the continuous improvement of treatment for life quality, the overall treatment costs are also increasing rapidly (8). The investigation and analysis of the economic burden of maintenance hemodialysis patients will provide a decision-making basis for the establishment of the medical system and the scope and standard of reimbursement of hemodialysis medical expenses, thereby improving the quality of medical insurance management in China, reducing the burden on patients, and improving the quality of life of patients $(9,10)$.

\section{The direct economic burden of maintenance hemodialysis patients}

\section{Drug costs}

The cost of drugs for hemodialysis patients mainly includes the cost of sanitary materials, Western medicine, and traditional Chinese medicine and herbal medicine, which accounts for about half of the total economic burden, constituting the core cost of treatment (11). Findings in this study also found that the cost of drugs was the highest in the resident medical insurance group, followed by the employee medical insurance group, the resident medical insurance + poverty relief group, and the employee medical insurance + poverty relief group, indicating that medical insurance for residents and employees has little effect on reducing the burden of drug costs for patients; comparatively, the national strategy of poverty relief has effectively reduced the burden of drug costs on patients. Naalweh (12) and Gulin (13) et al. have reported that this strategy of relief provides cheaper medical expenses for hemodialysis patients, and the cost of drugs has gradually replaced the cost of dialysis as the most major part of the direct economic burden. The country's poverty relief policy enables families with poor economic conditions to relieve economic pressure.

\section{Hospitalization costs}

Hospitalization costs involve a wide range of aspects, mainly hospital bed charges, temperature adjustment fees, examination fees, consultation fees, treatment fees, injection fees, blood transfusion fees, surgery fees, oxygen transfer fees, radiation fees, hyperbaric oxygen treatment fees, and graphic printing fees. The results of this study indicate that the hospitalization costs of patients in the employee medical insurance group were the highest, and the hospitalization costs of patients in the resident medical insurance + poverty relief group were significantly lower than those in the employee medical insurance group, which further confirms that the strategy of relief can better reduce the burden to families with maintenance hemodialysis patients (14).

\section{Dialysis and laboratory test costs}

The costs of dialysis and laboratory tests for patients are mainly included into the direct costs of patients during dialysis and the laboratory tests of patients (15). The results in this study showed that the comparison of costs of dialysis and laboratory tests of patients in different medical insurance groups were not significantly different $(\mathrm{P}>0.05)$. However, the average annual costs of dialysis for hemodialysis patients was significantly higher than the cost of hospitalization. Therefore, to reduce the direct economic burden of hemodialysis patients, government and related departments should focus on decreasing or alleviating drug and dialysis costs.

\section{The indirect economic burden of maintenance hemodialysis patients}

With the rapid development of the economy and social well-being of China, the value of workers has gradually increased; the indirect economic burden of maintenance hemodialysis patients is directly related to the decrease in income due to loss of work of the patients and their family members affected by the disease. Therefore, the indirect economic burden is also playing an increasingly important role in the total economic burden of patients. Previous studies have shown (16) that the indirect economic burden of patients did not exceed 8,000 CNY, which is less than $8 \%$ of the average annual total economic burden. However, in recent years, related studies have shown that the indirect economic burden has accounted for $15 \%$ of the average annual total economic burden (17). The results in this study showed that the lost average annual indirect income of patients and their families caused by this disease was 
15,604.20 $\pm 2,755.59$ CNY. The indirect economic burden of patients in the employee + resident medical insurance group was the greatest, which may be related to the income loss of the patients and their families. The higher the income earned, the greater the damage of the indirect economic burden. For patients eligible for national relief, the indirect economic burden was slightly affected due to their limited incomes.

\section{Related risk factors affecting the average annual total economic burden of maintenance hemodialysis patients}

The results of this study also found that factors such as age, occupation, monthly family income per capita, and medical insurance type were all risk factors that adversely affect the average annual economic burden of maintenance hemodialysis patients $(\mathrm{P}<0.01)$. The economic burden of older patients was higher than that of younger patients, mainly due to the fact that for different age groups, the medical insurance methods and reimbursement ratios differ. Secondly, the medical resources consumed and extent of medical care vary considerably depending on the specific disease conditions and this subsequently result in varying economic burdens $(18,19)$. Therefore, it is recommended that the government and related departments provide financial assistance to elderly patients and patients with low family incomes to reduce their financial burden.

This study also found that the economic burden of unemployed patients was significantly higher than that of employed patients, which may be due to the essential difference in the types of medical insurance between these two groups of patients, leading to different proportions in their reimbursement expenses. Compared with employed patients, unemployed patients do not receive sufficient employee medical insurance, resulting in a low reimbursement rate and a heavy financial burden. In addition, the indirect economic burden of unemployed patients was relatively heavy. Due to the impact of the disease, the patients and their families cannot fully participate in work, if at all, which indirectly causes a considerable decrease in economic income (20). Therefore, it is recommended that the government and relevant departments should further implement reasonable medical insurance methods for unemployed persons, which can effectively solve the core problem of difficult and expensive medical treatment for unemployed patients. In addition, due to the sample size and time problem, this group of research did not study the relationship between nursing, psychological aspects and influencing factors, which can be further explored with larger sample size.

In summary, various medical insurance systems can effectively reduce the financial burden of hemodialysis patients, but patients must still bear a relatively heavy financial burden. Therefore, it is necessary to further improve the medical insurance for hemodialysis patients, increase management efforts, and broaden the poverty relief policy.

\section{Acknowledgments}

Funding: None.

\section{Footnote}

Reporting Checklist: The authors have completed the MDAR reporting checklist. Available at http://dx.doi.org/10.21037/ apm-20-1787

Data Sharing Statement: Available at http://dx.doi. org/10.21037/apm-20-1787

Conflicts of Interest: All authors have completed the ICMJE uniform disclosure form (available at http://dx.doi. org/10.21037/apm-20-1787). The authors have no conflicts of interest to declare.

Ethical Statement: The authors are accountable for all aspects of the work in ensuring that questions related to the accuracy or integrity of any part of the work are appropriately investigated and resolved. This study was approved by Affiliated Hospital of North Sichuan Medical College (No. 2020ER(N)050-1). All procedures performed in this study involving human participants were in accordance with the Declaration of Helsinki (as revised in 2013). Informed consent was taken from all the patients.

Open Access Statement: This is an Open Access article distributed in accordance with the Creative Commons Attribution-NonCommercial-NoDerivs 4.0 International License (CC BY-NC-ND 4.0), which permits the noncommercial replication and distribution of the article with the strict proviso that no changes or edits are made and the original work is properly cited (including links to both the formal publication through the relevant DOI and the license). See: https://creativecommons.org/licenses/by-nc-nd/4.0/. 


\section{References}

1. Huang H, Woodruff TJ, Baer RJ, et al. Investigation of association between environmental and socioeconomic factors and preterm birth in California. Environ Int 2018;121:1066-78.

2. Seefried L, Genest F, Luksche N, et al. Efficacy and safety of whole body vibration in maintenance hemodialysis patients - A pilot study. J Musculoskelet Neuronal Interact 2017;17:268-74.

3. Drew DA, Koo BB, Bhadelia R, et al. White matter damage in maintenance hemodialysis patients: a diffusion tensor imaging study. BMC Nephrol 2017;18:213.

4. Zhang L, Luo H, Kang G, et al. The association between physical activity and mortality among patients undergoing maintenance hemodialysis. Int J Nurs Pract 2017. doi: 10.1111/ijn.12505.

5. Lu J, Zhu M, Liu S, et al. The relationship between survival rate and intradialytic blood pressure changes in maintenance hemodialysis patients. Ren Fail 2017;39:417-22.

6. Jinjuvadia R, Salami A, Lenhart A, et al. Hepatocellular Carcinoma: A Decade of Hospitalizations and Financial Burden in the United States. Am J Med Sci 2017;354:362-9.

7. Bakkal H, Dizdar OS, Uludag K. PT10.4: Association of handgrip strength with malnutrition inflammation score in maintenance hemodialysis patients. Clinical Nutrition 2019;38:55.

8. Iwata T, Mori T. Long-term Clinical Outcomes of Elective Carotid Artery Stenting in Patients Undergoing Maintenance Hemodialysis. Intern Med 2020;59:479-83.

9. Bradley CJ. Cancer, Financial Burden, and Medicare Beneficiaries. J Clin Oncol 2017;35:2461-2.

10. Tallman DA, Latifi E, Kaur D, et al. Dietary patterns and health outcomes among African American maintenance hemodialysis patients. Nutrients 2020;12:797.

11. Sasaki S, Hasegawa T, Kawarazaki H, et al. Correction: Development and Validation of a Clinical Prediction Rule

Cite this article as: $\mathrm{Ma} \mathrm{Y}$, Yu H, Sun H, Li M, Li L, Qin M. Economic burden of maintenance hemodialysis patients' families in Nanchong and its influencing factors. Ann Palliat Med 2020;9(6):3877-3884. doi: 10.21037/apm-20-1787 for Bacteremia among Maintenance Hemodialysis Patients in Outpatient Settings. PLoS One 2017;12:e0181800.

12. Naalweh KS, Barakat MA, Sweileh MW, et al. Treatment adherence and perception in patients on maintenance hemodialysis: a cross - sectional study from Palestine. BMC Nephrol 2017;18:178.

13. Gulin M, Klarić D, Ilić M, et al. Blood Pressure of Maintenance Hemodialysis Patients in the Dalmatian Region of Croatia: Differences between Hospital and Outof-Hospital Dialysis Centers. Blood Purif 2017;44:110-21.

14. Chirakarnjanakorn S, Navaneethan SD, Francis GS, et al. Cardiovascular impact in patients undergoing maintenance hemodialysis: Clinical management considerations. Int J Cardiol 2017;232:12-23.

15. Kaihan AB, Hishida $M$, Imaizumi T, et al. Circulating levels of $\mathrm{CD} 34+$ cells predict long-term cardiovascular outcomes in patients on maintenance hemodialysis. PLoS One 2019;14:e0223390.

16. Rossignol P, Frimat L, Zannad F. The safety of mineralocorticoid antagonists in maintenance hemodialysis patients: two steps forward. Kidney Int 2019;95:747-9.

17. Hsieh HL, Hsu SC, Cheng HS, et al. The influence of atrial fibrillation on the mortality of incident ESRD patients undergoing maintenance hemodialysis. PLoS One 2020;15:e228405.

18. McIntyre CW, Salerno FR. Diagnosis and Treatment of Intradialytic Hypotension in Maintenance Hemodialysis Patients. Clin J Am Soc Nephrol 2018;13:486-9.

19. Howell M, Walker RC, Howard K. Cost Effectiveness of Dialysis Modalities: A Systematic Review of Economic Evaluations. Appl Health Econ Health Policy 2019;17:315-30.

20. Shimamura T, Yamashita S, Ryuujin S, et al. Hematogenous pleural infection caused by Achromobacter xylosoxidans in a patient undergoing maintenance hemodialysis. J Infect Chemother 2020;26:389-92.

(English Language Editor: J. Gray) 